\title{
Different iron-handling in inflamed small and large cholangiocytes and in small and large-duct type intrahepatic cholangiocarcinoma
}

\author{
Romina Mancinelli, ${ }^{1 *}$ Antimo Cutone, ${ }^{2 *}$ Luigi Rosa, ${ }^{3}$ Maria Stefania Lepanto, ${ }^{3}$ Paolo Onori, ${ }^{1}$ Luigi Pannarale, ${ }^{1}$ \\ Antonio Franchitto, ${ }^{1,4}$ Eugenio Gaudio, ${ }^{1}$ Piera Valenti ${ }^{3}$ \\ ${ }^{1}$ Department of Anatomical, Histological, Forensic Medicine and Orthopedic Sciences, Sapienza University of Rome \\ ${ }^{2}$ Department of Biosciences and Territory, University of Molise, Pesche (IS) \\ ${ }^{3}$ Department of Public Health and Infectious Diseases, Sapienza University of Rome \\ ${ }^{4}$ Eleonora Lorillard Spencer Cenci Foundation, Rome, Italy
}

*These authors contributed equally to this work

\begin{abstract}
Cholangiocarcinoma (CCA) represents the second most common primary hepatic malignancy and originates from the neoplastic transformation of the biliary cells. The intrahepatic subtype includes two morpho-molecular forms: large-duct type intrahepatic CCA (iCCA) and small-duct type iCCA. Iron is fundamental for the cellular processes, contributing in tumor development and progression. The aim of this study was to evaluate iron uptake, storage, and efflux proteins in both lipopolysaccharide-inflamed small and large cholangiocytes as well as in different iCCA subtypes. Our results show that, despite an increase in interleukin- 6 production by both small and large cholangiocytes, ferroportin (Fpn) was decreased only in small cholangiocytes, whereas transferrin receptor-1 (TfR1) and ferritin (Ftn) did not show any change. Differently from in vitro models, Fpn expression was increased in malignant cholangiocytes of small-duct type iCCA in comparison to large-duct type iCCA and peritumoral tissues. TfR1, Ftn and hepcidin were enhanced, even if at different extent, in both malignant cholangiocytes in comparison to the surrounding samples. Lactoferrin was higher in large-duct type iCCA in respect to small-duct type iCCA and peritumoral tissues. These findings show a different iron handling by inflamed small and large cholangiocytes, and small- and large-duct type iCCA. The difference in iron homeostasis by the iCCA subtypes may have implications for the tumor management.
\end{abstract}

Key words: Inflammation; cholangiocytes; cholangiocarcinoma; iron-proteins; ferroportin; hepcidin; lactoferrin.

Correspondence: Romina Mancinelli, Department of Anatomical, Histological, Forensic Medicine and Orthopedics Sciences, Sapienza Università of Rome, Via Alfonso Borelli 50, 00161 Rome, Italy.

Tel.+39.06.49918063 - Fax: +39.064.9918062. E-mail: romina.mancinelli@uniroma1.it

Contributions: RM, PV, EG, designed the study; AC, LR, MSL, conducted in vitro experiments; RM, performed in vivo experiments; RM, AC, LR, AF, LF, PO, analyzed the data and prepared the figures; RM, AC, LR, EG, PV, wrote the manuscript.

Conflict of interest: The authors declare no conflict of interest.

Funding: This project received financial support from "Progetti Medi di Ateneo 2017" of Prof. Paolo Onori and "Progetti piccoli di Ateneo 2018” of Prof. Romina Mancinelli, Sapienza University of Rome.

Ethical Approval: The study was conducted in accordance with the Helsinki Declaration of 1975; human tissues were obtained from patients submitted to surgical resection, after informed consent, at the Department of Gastroenterology of the Academic General Hospital Umberto I, Sapienza University of Rome.

\section{Patient consent for publication:}

Informed consent: The manuscript does not contain any individual person's data in any form. 


\section{Introduction}

Cholangiocytes are simple epithelial cells that line the intrahepatic biliary tree, a complex three-dimensional network of tubular canals of different diameters within the liver. ${ }^{1,2}$ Small and large cholangiocytes reside primarily in small and large intrahepatic bile ducts, respectively, with different functions and responses to injury. ${ }^{3}$ In the human biliary tree, cholangiocytes are damaged in a variety of diseases, namely cholangiopathies, which often cause advanced liver failure. ${ }^{4}$ It is well-known the role of cholangiocytes in liver regeneration when hepatocyte regrowth is compromised, whereas it is still under study the role of biliary cells in innate and adaptive immune responses. ${ }^{5}$

In recent years, the incidence of hepatocellular carcinoma (HCC) and cholangiocarcinoma (CCA) has increased. ${ }^{6}$ Particularly, CCA is a devastating adenocarcinoma arising from malignant transformation of cholangiocytes and represents a heterogeneous group of malignant neoplasms. ${ }^{7}$ They are anatomically classified as intrahepatic (iCCA) (arising proximal to the second order bile ducts), perihilar (pCCA) (arising between the second order bile ducts and the insertion of the cystic duct into the common bile duct) and distal extrahepatic (dCCA) (arising between the insertion of the cystic duct and the ampulla of Vater) ${ }^{8,9}$ In addition to this anatomical classification, other factors such as tumor growth pattern and the cell of origin provide alternative methods of classification that may better predict tumor invasion and progression. ${ }^{10,11}$ iCCA is characterized by two histological subtypes: originating either from small or large intrahepatic bile ducts. ${ }^{12,13}$ The first type is called small-duct type iCCA showing an almost exclusively mass-forming growth pattern, ${ }^{14,15}$ whereas the second type is a mucinous iCCA, called large-duct type and appears as massforming, periductal infiltrating or intraductal growing. ${ }^{16,17}$ In fact, according to the World Health Organization (WHO) classification, CCAs include a pure mucin-secreting and a mixed form. ${ }^{18}$ The large-duct type iCCAs arise from the mucin secreting epithelium lining large ducts formed by mature cells; whereas, the small-duct type originates from non mucin-secreting cells lining small bile ducts or canals of Hering formed by immature cholangiocytes..$^{19,20}$ This histological subdivision of iCCA is of great importance because the different cell of origin reflects the diversities in risk factors, molecular profile, clinical outcome and response to treatment.

Several efforts have been made to decipher the molecular mechanisms and the concurrent factors involved in cancer development and proliferation and, among these, iron represents an important risk factor. ${ }^{21}$.

Iron is an essential element for living cells as component of fundamental biological processes such as DNA replication and energy production. However, iron can also be toxic when present in excess because of its capacity to generate reactive oxygen species (ROS), which can result in macromolecule damage, tissue injury and organ failure. ${ }^{22}$ As iron is extremely insoluble, mammals have evolved different iron-binding proteins, such as lactoferrin (Lf), ferritin (Ftn) and transferrin (Tf), to favor, respectively, its absorption, storage and delivery. ${ }^{23}$ Moreover, different iron transport systems ensure iron to be sorted among tissues, blood and secretions in a safe manner. In particular, the interface between systemic and cellular iron homeostasis mainly involves the cellular iron acquisition by Tf-bound iron through Tf receptor 1 (TfR1)mediated endocytosis, whereas, only for particular iron-recycling cells, such as enterocytes, hepatocytes and macrophages, intracellular iron can be (re-)exported into the plasma by the iron export complex, involving a permease, namely ferroportin (Fpn), and a ferroxidase. ${ }^{23,24}$ Indeed, systemic iron homeostasis relies on the concerted action of peculiar cell types, i.e. enterocytes, macrophages and hepatocytes, able to handle iron in the way to quickly respond to physiological demand or to pathological stimuli and conditions, including inflammation and cancer. ${ }^{23,25,26}$ In inflammatory conditions, usually associated to cancer development and progression, intracellular iron load increases due to the inactivation of the iron efflux system, mainly related to the interleukin (IL)-6-mediated overexpression of hepcidin, a liver-secreted hormone responsible for Fpn degradation. ${ }^{23}$ In this scenario, intracellular iron overload assumes a synergic role in promoting carcinogenesis, enabling cancer cells to exploit aerobic metabolism for proliferation, migration, invasion of surrounding tissues and metastatization. Therefore, it is not surprising that expression profiles of most of the iron-handling proteins result altered in many different types of tumors..$^{27,28}$

Even if the liver is the central organ for iron homeostasis, little is known about iron and cholangiocytes in healthy and pathological states, especially regarding the two subpopulations of small and large cholangiocytes. Therefore, our aim was to understand whether the anomalous expression of the major proteins involved in iron homeostasis could play a role in CCA development and progression. The in vitro experiments were conducted in murine small and large cholangiocytes stimulated with LPS, of which the expression of Fpn, cytosolic Ftn, TfR1, and IL-6 have been evaluated. The in vivo study was conducted in iCCA and peritumoral noncancerous tissue, of which iron uptake (TfR-1 and Lf), iron storage (Ftn), and iron efflux (Fpn and hepcidin) proteins were analyzed.

\section{Materials and Methods}

\section{Materials}

Reagents were purchased from Abcam (Cambridge UK), Sigma (St. Louis, MO, USA) and DBA Italia srl (Milan, Italy) unless otherwise indicated. The mouse monoclonal Lf antibody was obtained from Abcam (ab10110), the rabbit polyclonal Ftn heavy chain (H-Ftn) antibody from Santa Cruz Biotechnology (Santa Cruz, CA, USA; sc-25617), the mouse monoclonal TfR1 antibody from Santa Cruz Biotechnology (sc-65882), the mouse monoclonal Fpn antibody was generously provided by T. Arvedson (Amgen), ${ }^{29}$ and the mouse monoclonal Hepcidin from Santa Cruz Biotechnology (sc-100277). The LSAB System-HRP used for IHC were purchased from Dako-Agilent (Santa Clara, CA, USA), while to develop the immunoreaction DAB + substrate chromogen system (Dako Agilent) was used. ELISA kit for IL-6 quantification was obtained from BioLegend, USA. In the end, Schiff's reagent for histology was purchased from Carlo Erba (Milan, Italy; \#477591).

\section{Cell lines}

In vitro studies were performed in immortalized small and large cholangiocyte lines, which display morphological and functional characteristic similar to that of freshly isolated small and large cholangiocytes (gifts from Prof. G. Alpini, Indiana University, School of Medicine, Indianapolis, IN, USA)..$^{20,30}$ The cell lines were maintained in minimum essential medium (Invitrogen, Carlsbad, CA, USA) containing 10\% heat-inactivated FBS, $1 \%$ penicillin and streptomycin, and $2 \mathrm{mmol} / \mathrm{L}$ l-glutamine at $37^{\circ} \mathrm{C}$ in a humidified $5 \% \mathrm{CO}_{2}$ incubator. We have previously shown that these cells display morphological (difference in size and transepithelial resistance), ${ }^{3}$ phenotypic (expression of cytokeratin-19), ${ }^{3}$ and functional features (cAMP response in large but not small cholangiocytes), ${ }^{3}$ similar to freshly isolated small and large mouse $^{31}$ and rat ${ }^{32}$ cholangiocytes. 


\section{Stimulation of cholangiocytes with LPS}

Small and large cholangiocytes were seeded in $25 \mathrm{~cm}^{2}$ flasks at a density of approximately $10^{6}$ cells $/ \mathrm{mL}$ in $5 \mathrm{~mL}$ of the specific medium for $48 \mathrm{~h}$, at $37^{\circ} \mathrm{C}$ in an atmosphere of $95 \%$ air and $5 \%$ $\mathrm{CO}_{2}$. After $48 \mathrm{~h}$, cells were washed twice with $5 \mathrm{~mL}$ of PBS without calcium and magnesium and treated with 1 or $100 \mu \mathrm{g} / \mathrm{mL}$ LPS from E. coli (InvivoGen, San Diego, CA, USA). The stimulations were carried out for $48 \mathrm{~h}$ in an atmosphere of $95 \%$ air and $5 \% \mathrm{CO}_{2}$, at $37^{\circ} \mathrm{C}$. After $48 \mathrm{~h}$ of incubation, the supernatants were harvested, aliquoted, and stored at $-80^{\circ} \mathrm{C}$ for cytokine quantitation. The cells were scraped in $2 \mathrm{~mL}$ of PBS containing $1 \mathrm{mM}$ phenylmethylsulfonyl fluoride (PMSF), pelleted by centrifugation at 2,500 $\times \mathrm{g}$ for $5 \mathrm{~min}$, and stored at $-80^{\circ} \mathrm{C}$ for protein analysis.

\section{Cytokine analysis}

Quantitation of IL-6 was performed on cell monolayer supernatants of small and large cholangiocytes by ELISA, using murine ELISA Max Deluxe Sets (BioLegend, San Diego, CA, USA).

\section{Western blots}

Small and large cholangiocytes (about $5 \times 10^{6}$ cells) were lysed in $300 \mu \mathrm{L}$ of lysis buffer ( $25 \mathrm{mM} 3$-morpholinopropane-1-sulfonic acid $\mathrm{pH} 7.4 / 150 \mathrm{mM} \mathrm{NaCl} / 1 \%$ Triton containing $1 \mathrm{mM}$ PMSF, 2 $\mu \mathrm{M}$ leupeptin, and $2 \mu \mathrm{M}$ pepstatin) in ice for $1 \mathrm{~h}$. Total protein content of samples was measured by Bradford assay. For SDS-PAGE, $20 \mu \mathrm{g}$ of total protein in SDS sample buffer containing 1,4-dithiothreitol, were heat-treated (except for Fpn) and loaded. For Western Blot analysis, primary antibodies were diluted in PBST at following concentration: anti-Fpn $(1: 10,000)$, anti-TfR1 $(1: 5,000)$, anti-H-Ftn $(1: 10,000)$ and anti-actin $(1: 10,000)$. After incubation with the appropriate secondary Horseradish Peroxidase-conjugated antibody, blots were developed with Enhanced Chemiluminescence (ECL Prime; GE Healthcare, Amersham, UK).

Protein bands were quantified by densitometry analysis, with Image $\mathrm{J}$ and proteins levels were normalized by $\beta$-actin.

\section{Human samples}

Liver samples of fifteen human iCCA and peritumoral noncancerous tissue were obtained from patients submitted to surgical resection (aged 50 to 83 years) with iCCA presenting as a single mass lesion within the liver, after informed consent, at the Department of Gastroenterology of the Academic General Hospital Umberto I, Sapienza University of Rome in accordance with the ethical standards and the Helsinki Declaration of 1975.

In particular, iCCA can arise at any point of the intrahepatic biliary tree, from the second-order bile ducts. Furthermore, iCCA can be divided into two main subtypes according to the histological level. Small bile duct iCCA originates from small intrahepatic bile ducts and with minimal mucin production. Large bile duct iCCA arises in large intrahepatic bile ducts and contains mucinproducing columnar tumor cells. ${ }^{17}$

\section{Light microscopy: morphologic study}

Human samples were immediately fixed in $10 \%$ buffered formalin and then dehydrated with alcohol, cleared in xylene and embedded in paraffin wax. Afterwards, the tissues were serially sectioned, obtaining 3- $\mu \mathrm{m}$-thick sections that were stained with Periodic Acid-Schiff staining system (PAS). This method is used for detection of glycogen and mucin in tissues, which were stained purple, while the nuclei were stained blue. Sections were deparaffinated and rehydrated. Following washing with PBS, they were incubated with $0.5 \%$ periodic acid solution for $5 \mathrm{~min}$, then stained with Schiff's reagent for $15 \mathrm{~min}$, followed by counterstaining with hematoxylin solution for $2 \mathrm{~min}$. All steps were performed at room temperature, and cells were rinsed with tap water after each step. The final morphological analysis has been performed using a light microscopy Leica Microsystems DM 4500.

\section{Light microscopy: immunohistochemistry}

As previously described, human specimens were immediately fixed, dehydrated with alcohol, cleared in xylene, embedded in paraffin wax, and sectioned. For immunohistochemistry (IHC), endogenous peroxidase activity was blocked by incubation in hydrogen peroxide (3\%). Sections were then incubated overnight at $4{ }^{\circ} \mathrm{C}$ with the different antibodies (Lf, Ftn, TfR1, Fpn and hepcidin). Samples were rinsed with $1 \mathrm{x}$ phosphate buffered saline (PBS), incubated with secondary biotinylated antibody and then with Streptavidin-HRP (LSAB+ System-HRP). Diaminobenzidine (DAB) was used as chromogen, and sections were counterstained with Hematoxylin. For all immunoreactions, negative controls were also included. Immunohistochemical observations were taken by a light microscopy Leica Microsystems DM 4500, with a camera JenoptikProgRes C10 Plus (Jena, Germany). In addition, all slides were scanned by a digital scanner (Aperio Scanscope CS System, Aperio Digital Pathology; Leica Biosystems, Milan, Italy) and processed by ImageScope software. We calculated the area occupied by Lf, Ftn, TfR, Fpn and hepcidin-positive cholangiocytes in the several iCCA and peritumoral samples. The quantifications were carried out by an image analysis algorithm and expressed as the percentage of the total area. ${ }^{33}$

\section{Statistical analysis}

All experiments were run at least in triplicate. Results are expressed as mean \pm SE. Statistical analysis was performed with GraphPadPrism and analysis of variance was used to compare quantitative data populations with normal distribution and equal variance.

\section{Results}

\section{Iron proteins' changes in small and large cholangio- cytes upon LPS treatment}

To induce a putative inflammatory response in both small and large cholangiocytes, the cultures were stimulated with 1 or $100 \mu \mathrm{g} / \mathrm{mL}$ LPS. As shown in Figure 1A, the levels of IL-6 significantly increased upon treatment with both 1 and $100 \mu \mathrm{g} / \mathrm{mL}$ of LPS compared to un-stimulated small or large cholangiocytes. In particular, the IL- 6 synthesized by small cells stimulated with 1 or $100 \mu \mathrm{g} / \mathrm{mL}$ LPS increased by 2.5 folds with respect to unstimulated ones. On the other hand, large cholangiocytes were more responsive to higher LPS concentration, resulting in an increase by 3.5 folds in IL-6 production. Concerning iron-handling proteins, only small cholangiocytes showed a significant downregulation in Fpn expression following $100 \mu \mathrm{g} / \mathrm{mL}$ LPS challenge (Figure 1B). Regarding the large sub-population, even no significant difference in Fpn levels was recorded for both inflammatory challenges, a decreasing trend was observed upon high dose LPS (Figure 1B). Concerning Ftn and TfR1, both small and large cholangiocytes did not show any significant difference in their expression patterns upon stimulation with both high and low LPS concentrations (Figure $1 \mathrm{C}, \mathrm{D}$ ).

\section{Tissue morphology and expression analysis of iron- related proteins in human $\mathrm{iCCA}$}

The morphological aspects of the different hepatic samples were evaluated by PAS (Figure 2 A-D). Through this staining we differentiated six large-duct type iCCA, PAS positive and nine 
samples as small-duct type iCCA, PAS negative. The architecture of the peritumoral tissues, both from large-duct and small-duct type iCCA, shows the typical hepatic aspect, with cords of hepatocytes, presence of portal spaces and enlargements of the sinusoidal network (Figure $2 \mathrm{~A}, \mathrm{C}$ ). The diffused purple color represents the physiologic amount of glycogen deposition in hepatocytes. The large-duct type iCCA sample changes totally with absence of the normal hepatic morphology, cholangiocytes form large gland-like structures, that are composed of cuboidal to cylindrical cells characterized by a most pronounced mucin production (largest biliary ducts PAS positivity) as evident with the widespread purple color (Figure 2B). The small-duct type iCCA subtype was characterized by areas of small tubular cord-like structures and irregular lumina, mainly formed by mucin-negative cholangiocytes (smallest biliary ducts PAS negative) (Figure 2D).

To assess whether iron proteins are altered in iCCA, we analyzed the expression of Fpn, hepcidin, TfR1, and Ftn through immunohistochemistry. We have evaluated and quantified the protein expressions in peritumoral and tumoral tissues. Fpn expression was found increased in large-duct type iCCA when compared to the surrounding tissues, whereas no significant difference was recorded in small-duct type iCCA (Figure 3A). Although Fpn increased in large-duct type, surprisingly, hepcidin expression was raised in both iCCAs compared to the peritumoral tissue (Figure $3 B$ ). Furthermore, the expressions of Ftn and TfR1 were significantly increased in small-duct type iCCA, the tumor arising from non-mucin-secreting cholangiocytes lining small immature bile ducts, compared to cholangiocytes in surrounding liver, while we found a moderate enhancement in large-duct type iCCA, the tumor deriving from the mucin-secreting epithelium lining the large differentiated ducts (Figure 3 C,D). Lastly, Lf immunopositivity has been found up-regulated in large-duct type iCCA compared to small-duct type iCCA and peritumoral tissue (Figure 3E). The quantitative values of Fpn, hepcidin, TfR-1, Ftn and Lf are reported in Table 1.

\section{Discussion}

Liver is one of the primary organs involved in the maintenance of iron homeostasis. In this respect, iron-handling by hepatocytes in physiological, inflamed and tumor states, has been extensively unveiled. ${ }^{34}$ Conversely, the role of iron-related proteins in physiopathological cholangiocytes remains largely unknown. Indeed, in the last decade, only few studies have investigated the dysregulation of iron metabolism in CCA models in vitro ${ }^{35}$ as well as in CCA patients. ${ }^{36}$ However, no data on the differential role of small and large cholangiocytes in iron-handling, in either physiological or pathological conditions, has been reported. Here, we show, in an in vitro model of immortalized small and large cholangiocytes challenged with LPS, that both sub-populations remain very poorly responsive to the inflammatory stimulation regarding the ironrelated proteins. In particular, though significant up-regulation of IL-6 was recorded for both small and large cells, no significant variation in the expression of Ftn and TfR1 was observed. Conversely, Fpn showed a significant downregulation only in small cholangiocytes upon high dose LPS (Figure 1B). Even if large cholangiocytes did not modulate Fpn synthesis under inflammatory stimuli (Figure 1B), the results found with small cholangiocytes are in agreement with most of the studies on iron proteins in inflamed models, all showing Fpn to be regulated upon inflammatory challenges. ${ }^{37-40}$ In particular, Fpn is downregulated in inflamed

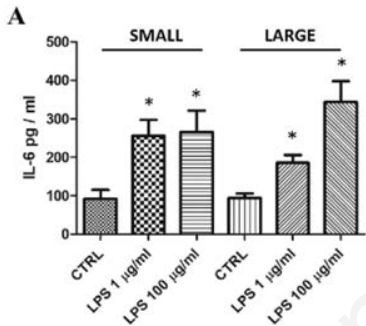

B

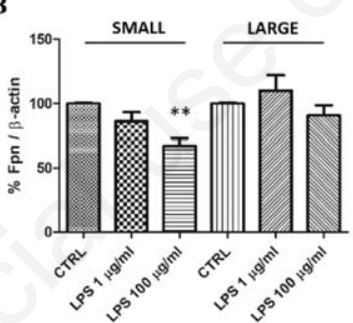

C

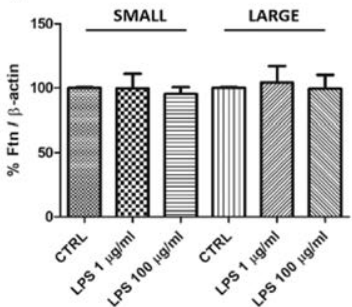

D
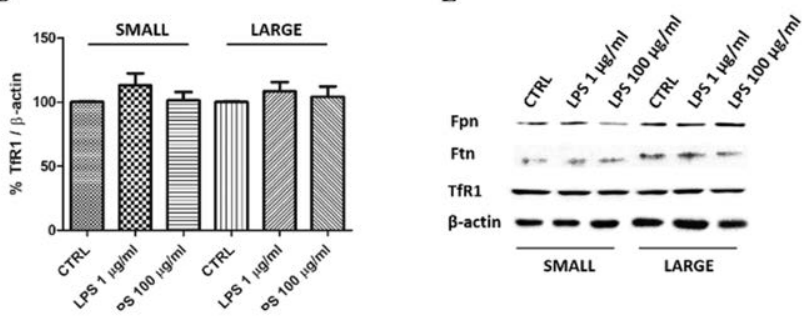

Figure 1. Changes in interleukin-6 (IL-6) (A), ferroportin (Fpn) (B), ferritin (Ftn) (C), and transferrin receptor 1 (TfR1) (D) levels in small and large cholangiocytes challenged with lipopolysaccharide (LPS) (1 and $100 \mu \mathrm{g} / \mathrm{mL}$ ). E) Representative Western blot. Each treatment was statistically compared to the related control condition (CTRL). Error bars: standard error of the mean; ${ }^{*} \mathrm{p}<0.05 ;{ }^{* *} \mathrm{p}<0.01$ (unpaired $t$-test).

Table 1. Measurement of the percentage of cholangiocytes positive for the expression of ferroportin (Fpn), hepcidin, transferrin receptor 1(TfR1), ferritin (Ftn) and lactoferrin (Lf), in the different liver sections.

\begin{tabular}{lccc} 
& Surrounding liver & Small-duct type iCCA & Surrounding liver \\
Fpn+ cholangiocytes (\%) & $19.1 \pm 1.6$ & $28.6 \pm 3.3^{\mathrm{a}}$ & $19.3 \pm 1.9$ \\
Hepcidin+ cholangiocytes (\%) & $16.1 \pm 2.0$ & $29.2 \pm 0.8^{\mathrm{a}}$ & $19.3 \pm 2.9$ \\
\hline TfR1+ cholangiocytes (\%) & $16.5 \pm 2.0$ & $41.2 \pm 1.0^{\mathrm{a}}$ & $3.9 \pm 2.5^{\mathrm{a}}$ \\
Ftn+ cholangiocytes (\%) & $18.4 \pm 1.3$ & $33.8 \pm 2.5^{\mathrm{a}}$ & $24.8 \pm 2.0^{\mathrm{a}}$ \\
\hline Lf+ cholangiocytes (\%) & $19.6 \pm 0.7$ & $17.8 \pm 1.7$ & $16.2 \pm 1.9$ \\
\hline
\end{tabular}

aP $<0.001$ (unpaired t-test) 
macrophages of in vitro models, ${ }^{37,38}$ whereas it is up-regulated in WT and cystic fibrosis (CF) bronchial epithelium both in vitro ${ }^{39}$ and murine model of Pseudomonas aeruginosa infection..$^{40}$ Therefore, it is emerging that Fpn can differently respond to infection or inflammation relating from its physiological role and from the in vitro or in vivo models. Indeed, Fpn acts as the only iron exporter from cell to blood in both hepatocytes and macrophages,${ }^{41}$ by allowing both body iron to be stored/redistributed and hemoglobin-deriving iron to be recycled, respectively. Following infection or inflammatory stimuli, IL-6 up-regulation, both through hepcidin-dependent ${ }^{41}$ or independent pathways, ${ }^{42,43}$ leads to Fpn downregulation, thus activating an unsafe intracellular iron overload which increases host susceptibility to infection ${ }^{23}$ or cancer proliferation. ${ }^{21,44}$ It is important to underline that the Fpn localization is also pivotal. As a matter of fact, in bronchial epithelium, Fpn, located on the cell apical side, acts as an iron detoxifying agent, able to translocate the metal in the bronchial lumen, thus allowing its further removal by the ciliary pathway. ${ }^{45}$ In this case, following infection/inflammatory stimuli Fpn is up-regulated, thus enhancing the activity of the detoxifying pathway thus facilitating the iron removal from bronchial lumen. ${ }^{40}$

Here, in our in vitro model, only small cholangiocytes seem to respond to inflammatory challenge, downregulating Fpn similarly
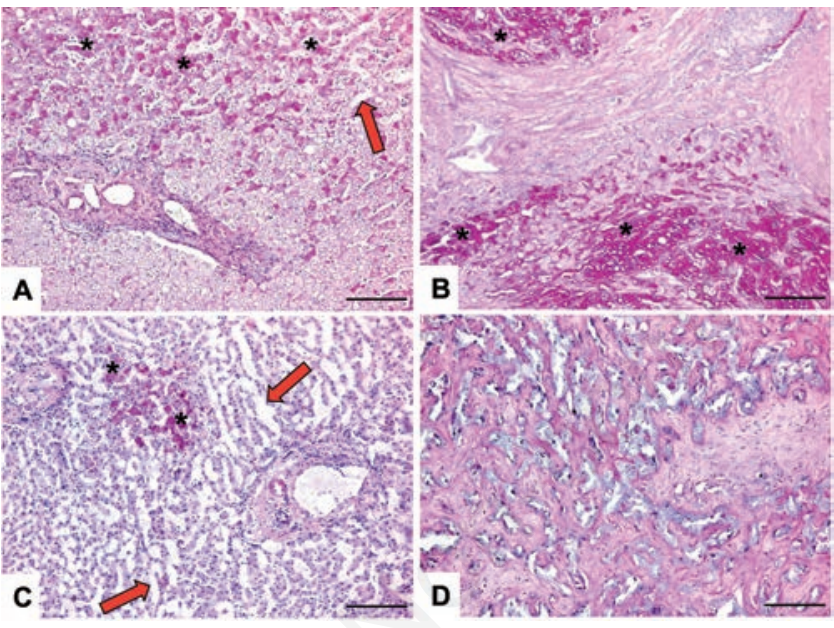

Figure 2. Representative images of Periodic-Acid Schiff (PAS) staining of peritumoral tissue and CCA. A) The image shows a peritumoral area from large-duct type iCCA; it presents a typical morphology with a portal space and the cords of hepatocytes greatly stained in dark pink/purple for the high percentage of carbohydrate content such as glycogen and mucin (black asterisks). B) Characteristic image of large-duct type iCCA stained with periodic-acid Schiff, in which the normal hepatic architecture is destroyed; these tumors are formed by acinar or tubular structures and tumor cells are columnar with abundant cytoplasm; as the name suggests, this subtype of tumor is mainly composed of mucin-producing cells, as shown by the diffused typical PAS color (black asterisks). C) An additional image of peritumoral tissue, in this case from small-duct type iCCA; there are not present relevant differences among the several peritumoral tissues, where the parenchyma could be characterized by enlargements of the sinusoids (red arrows). D) Periodic-acid Schiff stain in the other subtype of CCA: small-duct type iCCA; these tumors are formed by areas with small tubular cord-like structures with irregular cavities; the section shows histological differences compared to the mucin-subtypes mainly regarding the very few focal mucin producing regions. Scale bars: $100 \mu \mathrm{m}$. to that observed in inflamed macrophages and differently to that found in WT and CF bronchial epithelia where Fpn is localized on apical side. ${ }^{45}$ Even more analysis should be carried out to confirm the response of the two cholangiocyte sub-populations to inflammatory stimuli, our data on Fpn expression may suggest a potential different role between small and large cholangiocytes in iron-handling during infection/inflammation. Indeed, small cholangiocytes, probably due to their immature nature, may respond in a more efficient way in this iron-management when damaged, in comparison to the mature and well-differentiated large cholangiocytes.

In this study, we have also investigated the important role of iron acquisition, sequestration and export in contributing to the development and progression of biliary cancer. Several and recent studies have underlined that the iron homeostasis is dysregulated in cancer. ${ }^{46,47}$ In fact, iron is essential for normal cell function and many tumor cells require an increased, acquisition of iron to sustain growth and proliferation. ${ }^{44}$ Here, according to other studies, ${ }^{35,36}$ in cancer cells from both small-duct and large-duct type iCCA, we have observed an increased expression of TfR 1 to

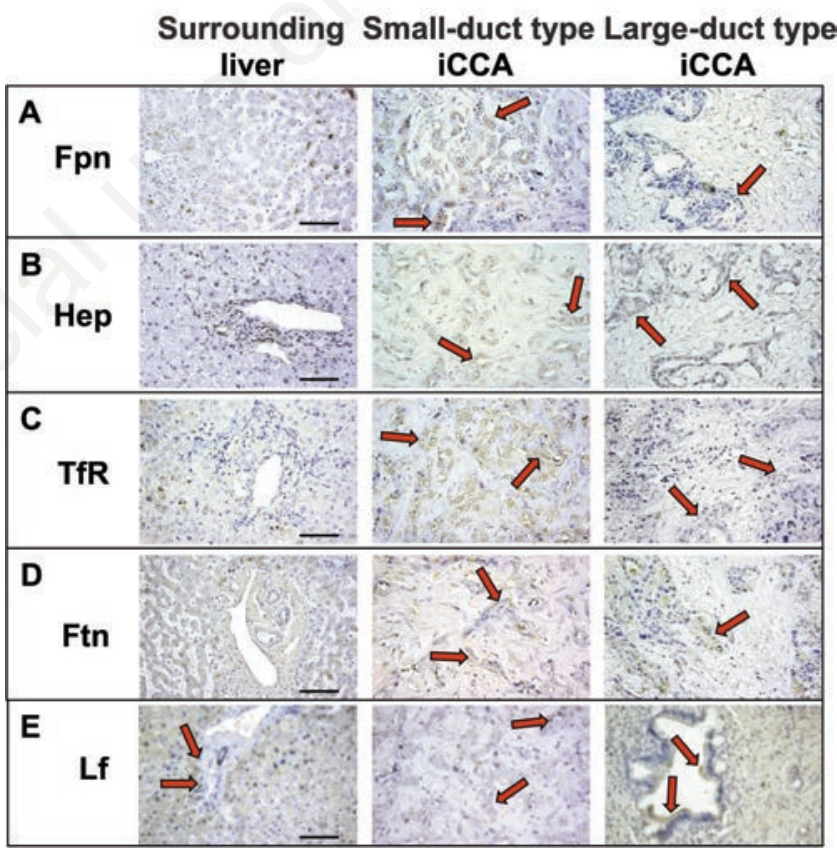

Figure 3. Representative images of the immunohistochemical study in both iCCA subtypes and peritumoral tissues. A) Immunohistochemistry for ferroportin (Fpn) (red arrows), in this case, small-duct type iCCA has showed a higher immunopositivity compared the peritumoral tissue and large-duct type iCCA. B) Immunohistochemistry for hepcidin (red arrows), tumoral biliary cells have displayed an important increase of the protein compared the peritumoral hepatic tissue. C) Immunohistochemistry for transferrin receptor protein (TfR1) (red arrows), cholangiocytes from small-duct type iCCA were intensively marked by the immunostaining compared the surrounding samples, while there was a lower increase in large-duct type iCCA always in respect to the peritumoral tissues. D) Immunohistochemistry for ferritin (Ftn) (red arrows), there was enhanced expression of the protein in tumoral cholangiocytes compared with the surrounding cholangiocytes. E) Immunohistochemistry for lactoferrin (Lf) (red arrows), showing an enhanced expression in large-duct type iCCA compared to peritumoral tissues (for quantitative analysis, see Table 1). Scale bars: $50 \mu \mathrm{m}$. 
enhance the intracellular iron availability. In relation to the increase of TfR1, we would have expected altered levels of Fpn, while we found similar expressions among peritumoral tissues and large-duct type iCCA $(19.3 \pm 1.9$ and $19.3 \pm 2.9)$, and a slight increase in small-duct type iCCA $(28.6 \pm 3.3)$, probably due to the poorly defined nature of the cells. In fact, small-duct type originates from small cholangiocytes, a population of immature cells, that in a specific position, such as the Hering's channels constitute the niche of hepatic progenitor cells (HPCs) able to differentiate into cholangiocytes and hepatocytes. Part of these cells could follow the hepatocyte lineage with a regular use of iron. Many tumor types exhibit an altered regulation of $\mathrm{Fpn}^{48}$ its overexpression could be linked to the inhibition of metastasis, as reported in lung and liver, through reduction of typical epithelial-to-mesenchymal transition markers such as Snail1, Twistl and vimentin. ${ }^{49}$ Thus, the different Fpn expression in the two iCCA subtypes could be linked to the progression of the damage, the small duct-type iCCA arising from an immature population of cells could induce different tumor outcomes. Moreover, Fpn overexpression in small-duct type iCCA, along with increased levels in TfR1 expression, could be related to increased iron-flux from the deeper ducts to the more superficial population of mucin-producing cells, which present TfR1 overexpression whereas basal level of Fpn. This may represent an intriguing mechanism through which small-cells could act as an iron gate able to furnish extra-demand of the metal to a more aggressive large-duct type iCCA. Regarding Ftn, in some tumors, it is present in low concentrations, but its upregulation has been described in several other tumoral tissues. ${ }^{50}$ In our samples, we found an enhancement of the Ftn expression in the two subtypes of iCCA compared with the peritumoral hepatic tissue, which is probably linked to its increase in stromal and inflammatory cells, hence suggesting its putative implication in counteracting oxidative stress in iron-overloaded cancer cells. ${ }^{50,51}$ Conversely, it has been reported how hepcidin levels increase in myeloma, renal cell carcinoma, prostate and other cancers for its central role in systemic iron uptake and recycling. ${ }^{52-54}$ Large-duct type iCCA $(30.9 \pm 2.5)$ and small-duct type iCCA (29.2 \pm 0.8$)$ exhibit higher levels of hepcidin expression compared the surrounding healthy liver in CCA patients $(16.7 \pm 2.2)$. Furthermore, hepcidin expression has been positively linked with tumor stage ${ }^{55}$ and it is activated by IL-6, the latter found up-regulated in CCA tissues. ${ }^{56}$ Increased IL-6 levels, typical in a tumoral situation, are also associated with an enhanced Lf, which is known to display a plethora of activities, including the inhibition of carcinogenesis in different tumors, like brain, breast, esophagus, lungs and liver, ${ }^{21,57-61}$ as well as the anti-inflammatory activity against IL-6 expression in vitro and in vivo. ${ }^{23,38,43,62-64} \mathrm{We}$ have previously showed that Lf and its receptors are present in biliary epithelium; ${ }^{65}$ we found an upregulation in experimental mouse models and in primary biliary cholangitis (PBC), suggesting an increased Lf uptake by damaged cholangiocytes. ${ }^{65}$ Our results show that Lf expression is higher in large-duct type iCCA compared with the small-duct type iCCA and the peritumoral tissues, suggesting high infiltration rates of neutrophils in the tumoral surrounding tissue and a possible supporting role of the Lf against the tumoral growth of large-duct type iCCA. The altered iron homeostasis shown here in small- and large-derived CCAs may be correlated to a global increase in iron flux and availability, underlined by elevated levels of Ftn and TfR1 in both subtypes of CCA as well as to normal or slight increased levels of Fpn in large-duct and smallduct type CCA, respectively.

In summary, although new experiments should be carried out and new markers evaluated, our data present novel peculiar aspects of iron-handling between small and large cholangiocytes and their associated iCCAs, showing different response to inflammatory stimuli and tumor-associated microenvironment. Indeed, iron-related proteins in immortalized cells seem to poorly respond to IL-6 signal- ing, whereas their expression is highly dysregulated in both CCAs, suggesting, once again, the primary role of iron in promoting tumoral growth and aggressiveness. It is also important to underline that the modulation of iron-proteins can be critically affected by the chosen model: in vitro or in vivo. Indeed, in vivo models for intrahepatic pathological states better reflect the complex biological interplay among different cell types, including cholangiocytes, hepatocytes and immune cells, which can cooperate in the establishing and maintenance of iron homeostasis disorders.

In conclusion, these data suggest that also in the intrahepatic $\mathrm{CCA}$ an altered iron homeostasis exists, which may play a role in aggressive CCA behavior. In particular, the different expressions of Fpn and Lf among large-duct type iCCA and small-duct type iCCA could be related to the activation of compensatory mechanisms used to antagonize tumoral progression and invasion. However, additional investigations will be needed to clarify the role of these proteins in biliary cancer biology and in the iron-regulatory pathway differently mediated in small and large-derived iCCA.

\section{Acknowledgments}

We thank Luiz Gabriel Dias Duarte Machado, Sapienza University of Rome, for his assistance with the revision of the manuscript and for his comments that improved the present article.

\section{References}

1. Glaser S, Gaudio E, Renzi A, Mancinelli R, Ueno Y, Venter J, et al. Knockout of the neurokinin-1 receptor reduces cholangiocyte proliferation in bile duct-ligated mice. Am J Physiol Gastrointest Liver Physiol 2011;301:G297-305.

2. Munshi MK, Priester S, Gaudio E, Yang F, Alpini G, Mancinelli R, et al. Regulation of biliary proliferation by neuroendocrine factors: implications for the pathogenesis of cholestatic liver diseases. Am J Pathol 2011;178:472-84.

3. Ueno Y, Alpini G, Yahagi K, Kanno N, Moritoki Y, Fukushima $\mathrm{K}$, et al. Evaluation of differential gene expression by microarray analysis in small and large cholangiocytes isolated from normal mice. Liver Int 2003;23:449-59.

4. Fava G, Demorrow S, Gaudio E, Franchitto A, Onori P, Carpino G, et al. Endothelin inhibits cholangiocarcinoma growth by a decrease in the vascular endothelial growth factor expression. Liver Int 2009;29:1031-42.

5. Banales JM, Huebert RC, Karlsen T, Strazzabosco M, LaRusso NF, Gores GJ. Cholangiocyte pathobiology. Nat Rev Gastroenterol Hepatol 2019;16:269-81.

6. Swenerton K, Jeffrey J, Stuart G, Roy M, Krepart G, Carmichael J, et al. Cisplatin-cyclophosphamide versus carboplatin-cyclophosphamide in advanced ovarian cancer: a randomized phase III study of the National Cancer Institute of Canada Clinical Trials Group. J Clin Oncol 1992;10:718-26.

7. Cardinale V, Renzi A, Carpino G, Torrice A, Bragazzi MC, Giuliante F, et al. Profiles of cancer stem cell subpopulations in cholangiocarcinomas. Am J Pathol 2015;185:1724-39.

8. Oliveira IS, Kilcoyne A, Everett JM, Mino-Kenudson M, Harisinghani MG, Ganesan K. Cholangiocarcinoma: classification, diagnosis, staging, imaging features, and management. Abdom Radiol (NY) 2017;42:1637-49.

9. Banales JM, Cardinale V, Carpino G, Marzioni M, Andersen J., Invernizzi P, et al. Expert consensus document: Cholangiocarcinoma: current knowledge and future perspectives consensus statement from the European Network for the Study of 
Cholangiocarcinoma (ENS-CCA). Nat Rev Gastroenterol Hepat 2016;13:261-80.

10. Bragazzi MC, Ridola L, Safarikia S, Matteo SD, Costantini D, Nevi L, et al. New insights into cholangiocarcinoma: multiple stems and related cell lineages of origin. Ann Gastroenterol 2018;31: 42-55.

11. Patel T. New insights into the molecular pathogenesis of intrahepatic cholangiocarcinoma. J Gastroenterol 2014; 49:165-72.

12. Aishima S Oda Y. Pathogenesis and classification of intrahepatic cholangiocarcinoma: different characters of perihilar large duct type versus peripheral small duct type. J Hepatobiliary Pancreat Sci 2015;22:94-100.

13. Mammola CL, Vetuschi A, Pannarale L, Sferra R, Mancinelli R. Epidermal growth factor-like domain multiple 7 (EGFL7): Expression and possible effect on biliary epithelium growth in cholangiocarcinoma. Eur J Histochem 2018;62:2971.

14. Liau JY, Tsai JH, Yuan RH, Chang CN, Lee HJ, Jeng YM. Morphological subclassification of intrahepatic cholangiocarcinoma: etiological, clinicopathological, and molecular features. Mod Pathol 2014;27:1163-73.

15. Kendall T, Verheij J, Gaudio E Evert M, Guido M, Goeppert B, et al. Anatomical, histomorphological and molecular classification of cholangiocarcinoma. Liver Int 2019;39: 7-18.

16. Cardinale V, Bragazzi MC, Carpino G, Torrice A, Fraveto A, Gentile R, et al. Cholangiocarcinoma: increasing burden of classifications. Hepatobiliary Surg Nut 2013;2:272-80.

17. Banales JM, Marin JJG, Lamarca A, Rodrigues PM, Khan SA, Roberts LR, et al. Cholangiocarcinoma 2020: the next horizon in mechanisms and management. Nat Rev Gastroenterol Hepatol 2020;17:557-88.

18. Komuta M, Govaere O, Vandecaveye V, Akiba J, Van Steenbergen W, Verslype C, et al. Histological diversity in cholangiocellular carcinoma reflects the different cholangiocyte phenotypes. Hepatology 2012;55:1876-88.

19. Brunt E, Aishima S, Clavien PA, Fowler K, Goodman Z, Gores $\mathrm{G}$, et al. cHCC-CCA: Consensus terminology for primary liver carcinomas with both hepatocytic and cholangiocytic differentation. Hepatology 2018;68:113-26.

20. Mancinelli R, Franchitto A, Glaser S, Meng F, Onori P, Demorrow $\mathrm{S}$, et al. GABA induces the differentiation of small into large cholangiocytes by activation of $\mathrm{Ca}(2+) / \mathrm{CaMK}$ Idependent adenylyl cyclase 8. Hepatology 2013;58:251-63.

21. Cutone A, Rosa L, Ianiro G, Lepanto MS, Bonaccorsi di Patti MC, Valenti $P$, et al. Lactoferrin's anti-cancer properties: Safety, selectivity, and wide range of action. Biomolecules 2020;10:456.

22. Andrews NC. Disorders of iron metabolism. N Engl J Med 1999;41:1986-95. Correction in: N Engl J Med 2000;342:364.

23. Rosa L, Cutone A, Lepanto MS, Paesano R, Valenti P. Lactoferrin: A natural glycoprotein involved in iron and inflammatory homeostasis. Int J Mol Sci 2017;18:1985.

24. Bonaccorsi di Patti, M.C, Cutone, A, Polticelli, F, Rosa, L, Lepanto, M.S, Valenti, P, et al. The ferroportin ceruloplasmin system and the mammalian iron homeostasis machine: Regulatory pathways and the role of lactoferrin. Biometals 2018, 31, 399-414.

25. Torti SV, Manz DH, Paul BT, Blanchette-Farra N, Torti FM. Iron and cancer. Annu Rev Nutr 2018;38:97-125.

26. Mancinelli R, Rosa L, Cutone A, Lepanto MS, Franchitto A, Onori P, et al. Viral hepatitis and iron dysregulation: Molecular pathways and the role of lactoferrin. Molecules 2020;25:E1997.

27. Kukulj S, Jaganjac M, Boranic M, Krizanac S, Santic Z, Poljak-Blazi M. Altered iron metabolism, inflammation, tran- sferrin receptors, and ferritin expression in non-small-cell lung cancer. Med Oncol 2010;27:268-77.

28. Wang Y, Yu L, Ding J, Chen Y. Iron metabolism in cancer. Int J Mol Sci 2018;20:E95.

29. Ross SL, Tran L, Winters A, Lee KJ, Plewa C, Foltz I, et al. Molecular mechanism of hepcidin-mediated ferroportin internalization requires ferroportin lysines, not tyrosines or JAKSTAT. Cell Metab 2012;15:905-17.

30. Alpini G, Glaser SS, Ueno Y, Pham L, Podila PV, Caligiuri A, et al. Heterogeneity of the proliferative capacity of rat cholangiocytes after bile duct ligation. Am J Physiol 1998;274:G767-75.

31. Glaser SS, Gaudio E, Rao A, Pierce LM, Onori P, Franchitto A, et al. Morphological and functional heterogeneity of the mouse intrahepatic biliary epithelium. Lab Invest 2009;89:456-69.

32. Alpini G, Roberts S, Kuntz SM, Ueno Y, Gubba S, Podila PV, et al. Morphological, molecular, and functional heterogeneity of cholangiocytes from normal rat liver. Gastroenterology 1996;110:1636-43.

33. Carpino G, Cardinale V, Renzi A, Hov JR, Berloco PB, Rossi $\mathrm{M}$, et al. Activation of biliary tree stem cells within peribiliary glands in primary sclerosing cholangitis. J Hepatol 2015;63:1220-8.

34. Wang CY, Babitt JL. Liver iron sensing and body iron homeostasis. Blood 2019;133:18-29.

35. Raggi C, Gammella E, Correnti M, Buratti P, Forti E, Andersen JB, et al. Dysregulation of iron metabolism in cholangiocarcinoma stem-like cells. Sci Rep 2017;7:17667.

36. Jamnongkan W, Thanan R, Techasen A, Namwat N, Loilome $\mathrm{W}$, Intarawichian $\mathrm{P}$, et al. Upregulation of transferrin receptor1 induces cholangiocarcinoma progression via induction of labile iron pool. Tumour Biol 2017;39:1010428317717655.

37. Cutone A, Frioni A, Berlutti F, Valenti P, Musci G, Bonaccorsi di Patti MC. Lactoferrin prevents LPS-induced decrease of the iron exporter ferroportin in human monocytes/macrophages. Biometals 2014;27:807-13.

38. Cutone A, Rosa L, Lepanto MS, Scotti MJ, Berlutti F, Bonaccorsi di Patti MC, et al. Lactoferrin efficiently counteracts the inflammation-induced changes of the iron homeostasis system in macrophages. Front Immunol 2017;8:705.

39. Frioni A, Conte MP, Cutone A, Longhi C, Musci G, Bonaccorsi di Patti MC, et al. Lactoferrin differently modulates the inflammatory response in epithelial models mimicking human inflammatory and infectious diseases. Biometals 2014;27:843-56.

40. Cutone A, Lepanto MS, Rosa L, Scotti MJ, Rossi A, Ranucci $\mathrm{S}$, et al. Aerosolized bovine lactoferrin counteracts infection, inflammation and iron dysbalance in a cystic fibrosis mouse model of Pseudomonas aeruginosa chronic lung infection. Int J Mol Sci 2019;20:2128.

41. Ganz T. Systemic iron homeostasis. Physiol Rev 2013;93:1721-41.

42. Guida C, Altamura S, Klein FA, Galy B, Boutros M, Ulmer AJ, et al. A novel inflammatory pathway mediating rapid hepcidinindependent hypoferremia. Blood 2015;125:2265-75.

43. Lepanto MS, Rosa L, Cutone A, Conte MP, Paesano R, Valenti P. Efficacy of lactoferrin oral administration in the treatment of anemia and anemia of inflammation in pregnant and non-pregnant women: An interventional study. Front Immunol 2018;9:2123.

44. Cairo G, Bernuzzi F, Recalcati S. A precious metal: Iron, an essential nutrient for all cells. Genes Nutr 2006;1:25-39.

45. Ghio AJ, Roggli VL, Soukup JM, Richards JH, Randell SH, Muhlebach MS. Iron accumulates in the lavage and explanted lungs of cystic fibrosis patients. J Cyst Fibros 2013;12:390-8. 46. Lobello N, Biamonte F, Pisanu ME, Faniello MC, Jakopin Ž, 
Chiarella E, et al. Ferritin heavy chain is a negative regulator of ovarian cancer stem cell expansion and epithelial to mesenchymal transition. Oncotarget 2016;7:62019-33.

47. Zhou Q, Chen J, Feng J, Wang J. E4BP4 promotes thyroid cancer proliferation by modulating iron homeostasis through repression of hepcidin. Cell Death Dis 2018;9:987.

48. Chen, Y, Zhang, S, Wang, X, Guo, W, Wang, L, Zhang, D, et al. Disordered signaling governing ferroportin transcription favors breast cancer growth. Cell. Signal. 2015, 27, 168-176.

49. Guo W, Zhang S, Chen Y, Zhang D, Yuan L, Cong H, et al. An important role of the hepcidin-ferroportin signaling in affecting tumor growth and metastasis. Acta Biochim Biophys Sin (Shanghai) 2015;47:703-15.

50. Torti SV, Torti FM. Iron and cancer: more ore to be mined. Nat Rev Cancer 2013;13:342-55.

51. Shpyleva SI, Tryndyak VP, Kovalchuk O, Starlard-Davenport A, Chekhun VF, et al. Role of ferritin alterations in human breast cancer cells. Breast Cancer Res Treat 2011, 126, 63-71.

52. Ganz T, Olbina G, Girelli D, Nemeth E, Westerman M. Immunoassay for human serum hepcidin. Blood 2008;112: 4292-7.

53. Tanno T, Rabel A, Alleyne M, Lee YT, Dahut WL, Gulley JL, et al. Hepcidin, anaemia, and prostate cancer. BJU Int 2011;107:678-9.

54. Kamai T, Tomosugi N, Abe H, Arai K, Yoshida K. Increased serum hepcidin-25 level and increased tumor expression of hepcidin mRNA are associated with metastasis of renal cell carcinoma. BMC Cancer 2009;9:270.

55. Xiang-Tao P. Expression of hepcidin and neogenin in colorectal cancer. Open Med (Wars) 2017;12:184-8.

56. Bei L, Lijie M, Wenqing T, Peixin H, Biwei Y, Lingxiao W, et al. FXR acts as a metastasis suppressor in intrahepatic cholangiocarcinoma by inhibiting IL-6-induced epithelial-mesenchymal transition. Cell Physiol Biochem 2018;48:158-72.

57. Cutone A, Colella B, Pagliaro A, Rosa L, Lepanto MS,
Bonaccorsi di Patti MC, et al. Native and iron-saturated bovine lactoferrin differently hinder migration in a model of human glioblastoma by reverting epithelial-to-mesenchymal transition-like process and inhibiting interleukin-6/STAT3 axis. Cell Signal 2020;65:109461.

58. Duarte DC, Nicolau A, Teixeira JA, Rodrigues LR. The effect of bovine milk lactoferrin on human breast cancer cell lines. J Dairy Sci 2011;94:66-76.

59. Fujita K, Ohnishi T, Sekine K, Iigo M, Tsuda H. Down-regulation of 2-amino-3,8-dimethylimidazo[4,5-f]quinoxaline (MeIQx)-induced CYP1A2 expression is associated with bovine lactoferrin inhibition of MeIQx-induced liver and colon carcinogenesis in rats. Jpn J Cancer Res 2002;93:616-25.

60. Tanaka T, Kawabata K, Kohno H, Honjo S, Murakami M, Ota T, Tsuda H. Chemopreventive effect of bovine lactoferrin on 4nitroquinoline 1-oxide-induced tongue carcinogenesis in male F344 rats. Jpn J Cancer Res 2000;91:25-33.

61. Chekhun VF, Storchai DM, Todor IN, Borikun TV, Lukianova N.Y. Antitumor and genotoxic effects of lactoferrin in Walker256 tumor-bearing rats. Exp Oncol 2018;40:200-4.

62. Valenti P, Frioni A, Rossi A, Ranucci S, De Fino I, Cutone A, et al. Aerosolized bovine lactoferrin reduces neutrophils and pro-inflammatory cytokines in mouse models of Pseudomonas aeruginosa lung infections. Biochem.Cell Biol 2017;95:41-7.

63. Lepanto, M.S, Rosa, L, Paesano, P, Valenti, P, Cutone, A. Lactoferrin in aseptic and septic inflammation. Molecules 2019;24:1323.

64. Rosa L, Lepanto MS, Cutone A, Siciliano RA, Paesano R, Costi R, et al. Influence of oral administration mode on the efficacy of commercial bovine lactoferrin against iron and inflammatory homeostasis disorders. Biometals 2020;33:159-68.

65. Mancinelli R, Olivero F, Carpino G, Overi D, Rosa L, Lepanto MS, et al. Role of lactoferrin and its receptors on biliary epithelium. Biometals 2018;31:369-79.

Received for publication: 25 June 2020. Accepted for publication: 14 September 2020.

This work is licensed under a Creative Commons Attribution-NonCommercial 4.0 International License (CC BY-NC 4.0).

(C) Copyright: the Author(s), 2020

Licensee PAGEPress, Italy

European Journal of Histochemistry 2020; 64:3156

doi:10.4081/ejh.2020.3156 